\title{
Rastreamento da frequência de comportamentos sugestivos de transtornos alimentares na Universidade Positivo
}

\section{Screening of the frequency of behaviors suggestive of eating disorders at Positivo University}

\author{
Rafael Pires ${ }^{1}$, José Pinto', Guilherme Santos ${ }^{1}$, Simoney Santos ${ }^{1}$, \\ Hicham Zraik', Lucas Torres ${ }^{1}$, Moacir Ramos ${ }^{2}$
}

Pires R, Pinto J, Santos G, Santos S, Zraik H, Torres L, Ramos M. Rastreamento da freqüência de comportamentos sugestivos de transtornos alimentares na Universidade Positivo. Rev Medicina (São Paulo). 2010 abr.-jun.;89(2):115-23.

\begin{abstract}
RESUMO: Objetivo: Rastrear a frequência de comportamentos sugestivos de transtornos alimentares em amostra de alunos de cursos pré-selecionados por sorteio da Universidade Positivo e com isso comparar a prevalência desses distúrbios alimentares entre os cursos selecionados e entre homens e mulheres, e confrontar com dados da literatura mundial. Métodos: Este é um estudo transversal no qual foram aplicadas as auto-escalas Bite (Teste de investigação Bulímica de Edimburgh) e EAT-26 (Teste de Atitudes Alimentares) em 463 universitários dos cursos da área de Ciências Biológicas e da Saúde da Universidade Positivo, pré-selecionados através de sorteio. Resultados: De acordo com a auto-escala EAT-26, 23 alunos (5\%) apresentaram possíveis transtornos de alimentação, com predominância significativa do sexo feminino. E, segundo a auto-escala BITE, 17 estudantes (4\%) apresentaram um escore compatível com bulimia nervosa. Encontramos ainda, através da escala BITE-gravidade, 6 alunos (1\%) com alto grau de gravidade e 33 alunos $(7,3 \%)$ com escore sugestivo de estado clínico comprometido. Conclusões: Este estudo mostra uma frequência de transtornos alimentares na população estudada, assim como comportamentos alimentares inadequados, principalmente no sexo feminino, semelhante à encontrada na literatura. Concluímos que esses achados são relevantes para a clínica da faixa etária em estudo e que podem proporcionar o desenvolvimento de medidas assistenciais para portadores de transtornos alimentares, bem como futuros trabalhos em que poderemos compreender melhor os fatores de risco para o desenvolvimento de distúrbios da alimentação.
\end{abstract}

DESCRITORES: Anorexia; Bulimia; Transtornos da alimentação; Estudos transversais; Estudantes de ciências da saúde; Fatores de risco

1. Doutorandos do Curso de Medicina da Universidade Positivo, Curitiba, PR.

2. Professor da Disciplina de Saúde da Família do Curso de Medicina da Universidade Positivo, Curitiba, PR.

Endereço para correspondência: Rafael Pires. e-mail: rafaelsouzapires@ hotmail.com 


\section{INTRODUÇÃO}

$\mathrm{O}$ $s$ transtornos alimentares são entidades de grande importância médico-social, pois podem comprometer seriamente a saúde dos indivíduos sintomáticos. Atitudes de comportamento alimentar preocupantes e problemáticas relativas ao medo da obesidade e o ideal de se encaixar no perfil de imagem exposto pela mídia são comumente encontrados. Essas síndromes têm origem multifatorial, acometem principalmente adolescentes e mulheres jovens em idade reprodutiva e apresentam importante prevalência na população geral. Esses transtornos são divididos em duas categorias principais: anorexia nervosa (AN) e bulimia nervosa $(\mathrm{BN})^{1}$.

A anorexia nervosa pode ser definida como uma recusa sistemática em manter o peso mínimo normal adequado à idade e altura, acompanhada de uma perturbação no modo como o indivíduo vivencia seu peso e sua forma física. A anorexia nervosa tem complicações sérias associadas com a desnutrição, como: comprometimento cardiovascular, desidratação, distúrbios eletrolíticos, distúrbios na motilidade gastrointestinal, infertilidade, hipotermia e outras evidências de hipometabolismo ${ }^{2,3}$. Fisheret al. ${ }^{4}$ relata que a presença de amenorréia é uma característica importante da anorexia nervosa, e ela está associada a uma combinação de fatores como: disfunção hipotalâmica, estresse, exercício em excesso, e perda de peso e gordura.

Por outro lado, as características essenciais da bulimia nervosa consistem de episódios de hiperfagia seguidos de métodos compensatórios inadequados para evitar o ganho de peso. Ela se caracteriza ainda pela auto-avaliação influenciada pela forma e peso corporal. Sendo assim, o indivíduo apresenta medo de ficar acima do peso, reconhece que segue padrão alimentar anormal, mas não se sente capaz de controlar seu próprio comportamento alimentar e, dessa maneira, o transtorno pode se estender por muitos anos. Para qualificar o transtorno, a compulsão periódica e os comportamentos compensatórios inadequados devem ocorrer, em média, pelo menos duas vezes por semana por três meses ${ }^{5}$. O comportamento bulímico tem como principais complicações: distúrbios eletrolíticos, irritação e sangramento gástrico e esofágico, anormalidades intestinais, erosão do esmalte dental e aumento das glândulas parótidas. Bradicardia de repouso, hipotensão e diminuição da taxa metabólica são observados em alguns bulímicos, podendo refletir na diminuição da atividade do sistema nervoso simpático e do eixo tireoidiano ${ }^{2,4,6}$. Nos dois distúrbios, o peso e o formato corporal exercem influência na determinação da auto-estima dos pacientes, que via de regra encontra-se rebaixada ${ }^{7}$.

A prevalência de anorexia nervosa e bulimia nervosa têm alcançado altas proporções na população geral. De acordo com um grande número de autores, a prevalência desses transtornos, que fica entre $1 \%$ e $4 \%$, vem aumentando significativamente nos últimos anos $6,8,9$. Segundo a American Psychiatric Association $^{7}$, a prevalência de anorexia nervosa varia cerca de 0,3 a 3,7\% e a prevalência de bulimia nervosa é cerca de 1,1 a 4\%, ambas na população jovem feminina. Os homens também são acometidos, mas em proporções menores, representando apenas $10 \%$ dos casos de transtornos alimentares ${ }^{10}$. A maioria dos estudos estabelece uma prevalência de anorexia nervosa de um caso entre 100 garotas adolescentes, sendo as mulheres de classe social mais alta as que têm o maior risco ${ }^{11}$.

A prevalência de bulimia nervosa clinicamente significativa em estudantes universitárias é maior que a da comunidade de forma geral, segundo alguns estudos ${ }^{12,13,14}$. Halmi et al. ${ }^{15}$ observaram que $13 \%$ de 539 estudantes universitárias tinham sintomas de bulimia nervosa com os critérios do DSM-III, enquanto Hart e Ollendick ${ }^{16}$ verificaram que $5 \%$ de outras 234 estudantes universitárias apresentavam bulimia nervosa, e que a incidência de bulimia nervosa nesse grupo era cinco vezes maior que a de mulheres empregadas. Herzog et al. ${ }^{17}$ observaram que $15 \%$ de estudantes de Medicina já tinham apresentado história de distúrbios alimentares. O alto nível de estresse dos estudantes de áreas das Ciências Biológicas e da Saúde, em virtude de exagerada carga horária, atividades curriculares e extracurriculares, autocobrança, e por serem estudantes de cursos que estejam relacionados à alimentação, à nutrição e aos cuidados com a composição corporal, possivelmente contribuem também para a maior taxa de prevalência de distúrbios alimentares nessa população.

Devido à sua importância epidemiológica, urge ampliar esses estudos com a utilização de instrumentos específicos para seu rastreamento, para que o processo de intervenção e prevenção na população seja efetivo. Os questionários autopreenchíveis são recomendáveis pela facilidade de administração, eficiência e economia no rastreamento de transtornos alimentares na população ${ }^{18,19}$. Apresentam propriedades psicométricas adequadas e permitem aos respondentes revelarem um comportamento que, por constrangimento, poderia deixá-los relutante numa entrevista face a face com o entrevistador1.

Acredita-se atualmente que exista uma etiopatogenia multifatorial, com hipóteses da influência combinada da dinâmica familiar, do meio cultural e de 
aspectos da personalidade do indivíduo como fatores concorrentes, para a predisposição, instalação e manutenção dos distúrbios ${ }^{1}$. Ainda, o sentimento de negação da própria condição patológica, muitas vezes consequência de tabu existente em torno dos sintomas dos transtornos alimentares, leva essas síndromes a se estenderem por um longo período de tempo sem serem diagnosticadas, acarretando o aparecimento de comorbidades e agravos à saúde ${ }^{1}$.

Devido ao apresentado acima, a pesquisa tem por objetivo rastrear a frequência de comportamentos sugestivos de transtornos alimentares através dos questionários mundialmente validados EAT-26 e BITE em amostra de alunos de cursos pré-selecionados por sorteio da Universidade Positivo (entidade privada) e com isso comparar a prevalência desses distúrbios alimentares entre os cursos selecionados e entre homens e mulheres, e confrontar com dados da literatura mundial. Também, a pesquisa tem o intuito de demonstrar a necessidade de fornecer apoio psicológico e nutricional a esse grupo populacional, visto que existe grande possibilidade dessas alterações de comportamentos alimentares estarem relacionadas ao ambiente universitário no qual estão inseridos.

\section{METODOLOGIA}

Foi realizado um levantamento de frequência de distúrbios alimentares entre estudantes de nível superior.

Foram pesquisados estudantes da Universidade Positivo na cidade de Curitiba no Paraná, sendo sorteados cinco cursos entre os nove cursos do Núcleo de Ciências Biológicas e Saúde (Medicina, Fisioterapia, Nutrição, Odontologia, Psicologia, Ciências Biológicas, Educação Física e Enfermagem). E, dentre esses, foram sorteadas duas séries, sendo excluída a última série de cada curso para evitar viés, visto que os alunos dos últimos anos de cada curso geralmente têm aulas fora do campus ou se encontram em estágios de difícil acesso. A amostra sorteada foi composta por alunos dos seguintes cursos e séries: $1^{\mathrm{a}}$ e $3^{\mathrm{a}}$ série de Farmácia e Medicina, $1^{\mathrm{a}}$ e $2^{\mathrm{a}}$ série de Nutrição, $1^{\mathrm{a}}$ e $4^{\mathrm{a}}$ série de Psicologia e $2^{\mathrm{a}}$ e $3^{\mathrm{a}}$ série de Odontologia.

A escolha da amostra foi realizada para atingir representatividade entre estudantes de nível superior na faixa etária entre $18-25^{10}$ anos, a qual é a mais prevalente nos estudos pesquisados, e entre os cursos das áreas biológicas e de saúde entre os quais o tema seria de mais fácil compreensão.

O período de aplicação dos questionários ocorreu entre os meses de agosto e outubro de 2008.
Os pesquisadores apresentavam-se ao professor que estivesse na sala de aula e solicitavam quinze minutos de aula para a aplicação do questionário. Este era respondido individualmente, após breve explicação sobre o trabalho, em que foram expostos os objetivos da pesquisa. Os questionários eram depositados em um envelope colocado à frente da sala, garantindo-se, dessa forma, o anonimato.

Com o intuito de obter uma maior taxa de respondedores, foram escolhidas as aulas em que a frequência de estudantes fosse maior em cada semestre. Ressaltava-se que o preenchimento não era obrigatório. Foi realizado controle sobre o número de alunos matriculados $(n=505)$, sendo 363 alunas e 142 alunos. O número de questionários entregues foi $463(n=463$, sendo estes os alunos presentes no momento da aplicação do teste). Além do critério de presença, foram excluídos os questionários de alunos com idades diferentes da faixa etária do estudo e aqueles que assinalassem sim em uma questão formulada referente ao diagnóstico médico confirmado de doenças prévias (Diabetes Mellitus, câncer, doenças da tireóide, distúrbios psiquiátricos e doenças infecciosas crônicas) que viessem a interferir com a análise dos dados.

Foram excluídos 15 questionários. Destes, oito apresentavam idade superior a 25 anos, sete apresentavam doenças previamente diagnosticadas e nenhum questionário foi entregue incompleto, totalizando uma amostra de 448 questionários incluídos.

\section{Instrumentos}

O questionário utilizado foi de autopreenchimento e não continha a identificação do participante. Baseava-se em perguntas pertencentes às escalas validadas mais utilizadas nos estudos sobre transtornos alimentares, em suas respectivas versões em língua portuguesa.

São elas:

1. BITE - Teste de Investigação Bulímica de Edimburgo $^{20}$ - tradução de Cordás e Hochgraf ${ }^{21}$, que permite identificar comedores compulsivos e obter dados sobre aspectos cognitivos e comportamentais da bulimia nervosa;

2. EAT-26 - Teste de Atitudes Alimentares resumido ${ }^{22}$ - tradução de Nunes et al..$^{23}$, que avalia os riscos de se desenvolver comportamento e atitudes típicos de pacientes com anorexia nervosa.

$O$ resultado do BITE foi obtido pela escala de sintomas e pela escala de gravidade, que mede a gravidade do comportamento compulsivo pela freqüência. A classificação segundo o BITE é a seguinte: 


\section{Escala de sintomas}

1. Escore maior ou igual a 20: presença de comportamento alimentar compulsivo com grande possibilidade de preencher critérios diagnósticos para bulimia pelo DSM-III-R (APA, 1987).

2. Escore entre 10 e 19: sugestivo de padrão alimentar não usual. Em geral, não estão presentes todos os critérios para bulimia. Deve ser seguido de entrevista clínica. Escores de 15 a 19 devem ser obrigatoriamente seguidos de entrevista. Podem representar um grupo subclínico de indivíduos com alimentação compulsiva, bulímicos em estágio inicial ou bulímicos em recuperação.

3. Escore abaixo de 10: normalidade.

Escala de gravidade

1. Escore maior ou igual a 10: indica alto grau de gravidade. comprometido.

2. Escore maior que 5: indica estado clínico

A classificação segundo o EAT-26 foi:

1. Escore maior ou igual a 20: alto risco;

2. Escore de 10 a 19: baixo risco;

3. Escore de 0 a 9: fora de risco.

Foi atribuído valor positivo (EAT+) para EAT $>20$ e valor negativo (EAT -) para EAT < 20.
No Termo de Consentimento Livre e Esclarecido foi orientado que os participantes que apresentassem preocupação com os distúrbios alimentares poderiam procurar o ambulatório de Psicologia da Universidade Positivo, conforme contato realizado com a coordenação do curso de Psicologia. Além disso, os resultados deste serão apresentados para os cursos que participaram da pesquisa.

Os dados colhidos através do questionário foram codificados e armazenados em programa estatístico (Microsoft Excel 2007). A análise estatística básica para avaliação de variáveis qualitativas foi realizada pelo mesmo programa, utilizando-se o teste do qui-quadrado $\left(\mathrm{X}^{2}\right)$.

\section{RESULTADOS}

Entre os 448 questionários incluídos, obtevese a seguinte distribuição de estudantes por sexo: $322(71,8 \%)$ alunas e $126(28,2 \%)$ alunos; a média de faixa etária de todo o grupo foi de 19,7 anos, feminino e masculino.

As taxas de respondedores do questionário por turma estão apresentadas na Tabela 1, na qual se observa uma taxa global de respostas de $88,8 \%$.

TABELA 1: Proporção de alunos submetidos à avaliação sobre distúrbios alimentares na Universidade Positivo em 2008

\begin{tabular}{|c|c|c|c|c|}
\hline Curso (Série) & Total de alunos & $\begin{array}{c}\text { Questionários } \\
\text { entregues }\end{array}$ & $\begin{array}{c}\text { Questionários } \\
\text { avaliados }\end{array}$ & Frequência (\%) \\
\hline Medicina (1 $\left.1^{a}\right)$ & 57 & 54 & 50 & 87,7 \\
\hline Medicina $\left(3^{\underline{a}}\right)$ & 60 & 57 & 55 & 91,7 \\
\hline Farmácia $\left(1^{a}\right)$ & 60 & 55 & 53 & 88,3 \\
\hline Farmácia (2ª$)$ & 45 & 41 & 40 & 88,9 \\
\hline Odontologia (2 $\left.{ }^{\mathrm{a}}\right)$ & 58 & 52 & 52 & 89,7 \\
\hline 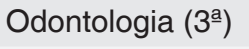 & 74 & 67 & 64 & 86,5 \\
\hline Nutrição $\left(1^{\text {a }}\right)$ & 29 & 25 & 25 & 86,2 \\
\hline Nutrição $\left(2^{\mathfrak{a}}\right)$ & 28 & 26 & 26 & 92,9 \\
\hline Psicologia (1aㅡ) & 54 & 51 & 49 & 90,7 \\
\hline Psicologia (4를 & 40 & 35 & 34 & 85,0 \\
\hline Total & 505 & 463 & 448 & 88,8 \\
\hline
\end{tabular}

Os escores médios do EAT-26, além do BITE, tanto escala de sintomas quanto gravidade, estão apesentados nas Tabelas 2,3 e 4, respectivamente, nas quais se evidencia uma prevalência global de anorexia nervosa pelo EAT-26 de $5 \%$, havendo predominância de diagnóstico entre mulheres (OR: 8,22; $p<0,05)$; uma prevalência global de sintomas sugestivos de bulimia de $4 \%$, havendo predomíno destes sintomas entre as alunas (OR: 2,$93 ; p<0,05$ ); uma prevalência global de gravidade de bulimia de $1 \%$, não havendo diferença significativa entre os sexos (OR: 1,96; $p>0,05$ ).

Em todas as análises dos escores utilizados na distribuição por faixa etária e entre os cursos, não foram encontradas diferenças significativas. 
TABELA 2: Proporção de estudantes com escores EAT superior ou igual a 20 da Universidade Positivo em 2008

\begin{tabular}{|c|c|c|c|c|}
\hline Curso & Sexo & Série & $\mathrm{N}^{\circ}$ de alunos & Score EAT \\
\hline \multirow{4}{*}{ Farmácia } & Masculino & 1 & 14 & $0(0 \%)$ \\
\hline & Masculino & 3 & 17 & $1(6 \%)$ \\
\hline & Feminino & 1 & 39 & $1(3 \%)$ \\
\hline & Feminino & 3 & 23 & $3(13 \%)$ \\
\hline \multirow{4}{*}{ Medicina } & Masculino & 1 & 25 & $0(0 \%)$ \\
\hline & Masculino & 3 & 30 & $0(0 \%)$ \\
\hline & Feminino & 1 & 25 & $4(16 \%)$ \\
\hline & Feminino & 3 & 25 & $2(8 \%)$ \\
\hline \multirow{2}{*}{ Nutrição } & Feminino & 1 & 25 & $2(8 \%)$ \\
\hline & Feminino & 2 & 26 & $0(0 \%)$ \\
\hline \multirow{4}{*}{ Odontologia } & Masculino & 2 & 12 & $0(0 \%)$ \\
\hline & Masculino & 3 & 20 & $0(0 \%)$ \\
\hline & Feminino & 2 & 40 & $2(5 \%)$ \\
\hline & Feminino & 3 & 44 & $3(7 \%)$ \\
\hline \multirow{4}{*}{ Psicologia } & Masculino & 1 & 7 & $0(0 \%)$ \\
\hline & Masculino & 4 & 6 & $0(0 \%)$ \\
\hline & Feminino & 1 & 42 & $4(10 \%)$ \\
\hline & Feminino & 4 & 28 & $1(4 \%)$ \\
\hline Total & & & 448 & $23(5,1 \%)$ \\
\hline
\end{tabular}

TABELA 3: Proporção de estudantes conforme a distribuição de escores bite escala de sintomas superior ou igual a 20 da Universidade Positivo em 2008

\begin{tabular}{llccc}
\hline Curso & Sexo & Série & No de alunos $^{*}$ Score Bite S \\
\hline \multirow{4}{*}{ Farmácia } & Masculino & 1 & 14 & $2(14 \%)$ \\
& Masculino & 3 & 17 & $0(0 \%)$ \\
& Feminino & 1 & 39 & $0(0 \%)$ \\
& Feminino & 3 & 23 & $2(9 \%)$ \\
Medicina & Masculino & 1 & 25 & $0(0 \%)$ \\
& Masculino & 3 & 30 & $0(0 \%)$ \\
& Feminino & 1 & 25 & $1(4 \%)$ \\
Nutrição & Feminino & 3 & 25 & $0(0 \%)$ \\
& Feminino & 1 & 25 & $2(8 \%)$ \\
& Feminino & 2 & 26 & $1(4 \%)$ \\
Odontologia & Masculino & 2 & 12 & $0(0 \%)$ \\
& Masculino & 3 & 20 & $0(0 \%)$ \\
& Feminino & 2 & 40 & $4(10 \%)$ \\
& Feminino & 3 & 44 & $2(5 \%)$ \\
Psicologia & Masculino & 1 & 7 & $0(0 \%)$ \\
& Masculino & 4 & 6 & $0(0 \%)$ \\
Total & Feminino & 1 & 42 & $1(2 \%)$ \\
\hline \multirow{5}{*}{ Feminino } & 4 & 28 & $2(7 \%)$ \\
& & & 448 & $17(3,8 \%)$ \\
\hline
\end{tabular}


Pires R, et al. Rastreamento da frequência de comportamentos de transtornos alimentares

TABELA 4: Proporção de estudantes conforme a distribuição de escores Bite escala de gravidade superior ou igual a 10 da Universidade Positivo em 2008

\begin{tabular}{|c|c|c|c|c|}
\hline Curso & Sexo & Série & $\mathrm{N}^{\circ}$ de alunos & Score Bite G \\
\hline \multirow{4}{*}{ Farmácia } & Masculino & 1 & 14 & $1(7 \%)$ \\
\hline & Masculino & 3 & 17 & $0(0 \%)$ \\
\hline & Feminino & 1 & 39 & $0(0 \%)$ \\
\hline & Feminino & 3 & 23 & $2(9 \%)$ \\
\hline \multirow{4}{*}{ Medicina } & Masculino & 1 & 25 & $0(0 \%)$ \\
\hline & Masculino & 3 & 30 & $0(0 \%)$ \\
\hline & Feminino & 1 & 25 & $0(0 \%)$ \\
\hline & Feminino & 3 & 25 & $0(0 \%)$ \\
\hline \multirow{2}{*}{ Nutrição } & Feminino & 1 & 25 & $1(4 \%)$ \\
\hline & Feminino & 2 & 26 & $0(0 \%)$ \\
\hline \multirow{4}{*}{ Odontologia } & Masculino & 2 & 12 & $0(0 \%)$ \\
\hline & Masculino & 3 & 20 & $0(0 \%)$ \\
\hline & Feminino & 2 & 40 & $1(3 \%)$ \\
\hline & Feminino & 3 & 44 & $0(0 \%)$ \\
\hline \multirow{4}{*}{ Psicologia } & Masculino & 1 & 7 & $0(0 \%)$ \\
\hline & Masculino & 4 & 6 & $0(0 \%)$ \\
\hline & Feminino & 1 & 42 & $1(2 \%)$ \\
\hline & Feminino & 4 & 28 & $0(0 \%)$ \\
\hline Total & & & 448 & $6(1,3 \%)$ \\
\hline
\end{tabular}

\section{DISCUSSÃO}

Os questionários EAT-26 e BITE vêm sendo utilizados em diversos países no rastreamento de comportamentos sugestivos de transtornos alimentares. Têm sido apontados como as escalas mais utilizadas nos estudos sobre os transtornos da alimentação ${ }^{24}$, apresentando boa acurácia no rastreamento dos mesmos ${ }^{25}$.

Bulimia nervosa e anorexia nervosa são desordens alimentares que geralmente se iniciam na adolescência, com um período de pico por volta dos $18-20$ anos de idade ${ }^{26}$. O presente estudo obteve uma idade média de 19,7 anos.

Apesar de o EAT-26, em estudos populacionais, apresentar um baixo valor preditivo para a anorexia nervosa (entre 100 pontuadores altos apenas 19 eram casos de anorexia nervosa), o instrumento pode ser utilizado como um índice da gravidade das preocupações típicas de pacientes com transtorno alimentar, principalmente a intenção de emagrecer e o medo de ganhar peso ${ }^{24}$. Dessa forma, pode-se apontar a necessidade de intervenção coletiva ou, nos casos mais sintomáticos, de encaminhamento aos cuidados de profissional especializado.

A freqüência de anorexia nervosa pelo EAT26 (maior ou igual a 20) encontrada no presente estudo foi de $5 \%$ (23 casos, sendo apenas um caso do sexo masculino), semelhante ao resultado obtido por Souza et al. ${ }^{27}$, o qual analisou universitárias da Universidade Federal do Ceará e encontrou frequência de $5,5 \%$ para anorexia nervosa. No entanto, em um estudo realizado em escolares por Vilela et al. ${ }^{28}$, encontrou-se frequencia de $13,3 \%$, mais expressiva que a frequência encontrada neste estudo.Outros estudos como o de Pinto et al. ${ }^{1}$, que analisaram apenas estudantes de medicina, observaram que cerca de $28 \%$ dos alunos possuiam risco elevado de apresentar transtornos alimentares, número mais expressivo que o encontrado pelo presente trabalho. 
Souza et al. ${ }^{27}$ sugerem que estudantes de escolas particulares poderiam apresentar taxas ainda mais altas de escores no teste EAT-26, por pertencerem à categoria econômica mais favorável e, assim, apresentarem um fator de risco para o desenvolvimento de transtornos alimentares. No entanto, não foram avaliados ainda sintomas de transtornos alimentares em estudantes de cursos universitários pertencentes a categorias econômicas menos favorecidas, para concluir que, realmente, estudantes de poder aquisitivo maior são mais suscetíveis a transtornos alimentares. Em trabalho realizado por Dunker et al. ${ }^{29}$, com adolescentes em escolas privadas e públicas, a porcentagem de score EAT-26 sugestivos de transtornos alimentares foi de $34,3 \%$,e $28,4 \%$ respectivamente, no entanto essa diferença não foi estatisticamente significante.

A freqüência de alunos com comportamento sugestivo de bulimia nervosa (BITE maior ou igual a 20 - escala de sintomas) neste estudo foi de $4 \%$ (17 casos, sendo 2 masculinos), semelhante à obtida por Souza et al. ${ }^{27}$; Drewnowski et al..$^{30}$; Katzman et al. ${ }^{31}$; Pyle et al. ${ }^{32}$ que foi de aproximadamente $4 \%$ entre estudantes universitárias. Em estudo feito com alunos de uma instituição pública de ensino superior em Ouro Preto-MG, constatou-se, através do BITE, que em 3\% da amostra estavam presentes sintomas de bulimia nervosa ${ }^{33}$. Nunes et al. ${ }^{34}$, avaliando 513 mulheres residentes na zona urbana de Porto AlegreRS, com idade entre 12 e 29 anos, verificaram que $3,3 \%$ apresentaram padrão alimentar severamente perturbado e compulsão para comer. Ghazal et al..$^{35}$, em uma pesquisa comparativa entre estudantes com idade entre 15 e 24 anos, de escolas marroquina e francesa, observaram que a prevalência de bulimia nervosa no grupo da escola francesa foi de 3,8\% entre as garotas, valor esse muito próximo ao encontrado no presente estudo. Da mesma forma, Miotto et al ${ }^{36}$, ao avaliarem transtornos alimentares e tendências suicidas em estudantes da Itália com idade entre 15 e 19 anos, encontraram prevalência de sintomas de bulimia nervosa em $4,1 \%$ das garotas avaliadas por meio do BITE.

Em estudo publicado por Mehler et al. ${ }^{26}$ foi demonstrada uma distribuição de ocorrências entre mulheres e homens de 10:1 a 20:1 e o índice encontrado no presente estudo foi de 3:1, demonstrando que a freqüência de bulimia nervosa entre mulheres e homens vem aumentando no decorrer das décadas.

Ainda em relação à escala BITE sintomas, foram encontrados 85 (19\%) alunos com escore entre 10-19 pontos, escore esse sugestivo de padrão alimentar não usual. No estudo na Faculdade de
Medicina da Universidade Federal do Ceará, o resultado encontrado foi de $23,1 \%$ de sintomas subclínicos agrupados como comportamentos alimentares não usuais ${ }^{27}$. Estudantes de 18 a 25 anos, de faculdade pública de Minas Gerais, foram investigados através do BITE, revelando-se que $16,4 \%$ dos estudantes apresentaram padrão alimentar pouco usual ${ }^{37}$. E no estudo de Cenci et al. ${ }^{5}$ o valor encontrado para a categoria comportamentos alimentares não usuais foi de $20,9 \%$. Esses estudos, juntamente com o presente estudo, demonstram que os valores sugestivos de padrão alimentar não usual é de 4 a 5 vezes maior que o padrão para valores sugestivos de bulimia nervosa.

Em relação à escala de gravidade do BITE, $6(1 \%)$ alunos tiveram escores maiores ou iguais a 10 , o que indica alto grau de gravidade e $33(7,3 \%)$ alunos apresentaram escores entre 5 a 9 , sugestivo de estado clínico comprometido.

Apesar de terem sido analisados e cruzados os escores dos questionários, não houve diferenças significativas entre os cursos analisados. No entanto, foi observado que a presença dos transtornos alimentares foi significativamente maior em mulheres.

O tema ainda é muito pouco estudado; de acordo com a literatura, estudos sobre prevalência de transtornos alimentares ainda são escassos no Brasil. Com a boa taxa de resposta obtida no estudo, a utilização de questionários validados e a forma de seleção da amostra contribuíram para a validade interna do estudo, diminuindo a chance de ocorrência de erros sistemáticos.

Por fim, acreditamos que a importância deste trabalho consiste no fato de ser uma das primeiras publicações abordando os transtornos alimentares em universitários de cursos de Ciências Biológicas e da Saúde no sul do Brasil, e pelo fato de demonstrar, assim como a literatura internacional, que os transtornos alimentares estão em relativa ascensão em todo o mundo. São imprescindíveis mais investigações sobre esta temática, já que os resultados obtidos até agora evidenciam que nas populações jovens dos países em desenvolvimento existe uma alta prevalência de transtornos alimentares em potencial, condição semelhante à encontrada na população de países considerados desenvolvidos. Pesquisas na área são extremamente relevantes, pois podem contribuir à busca de soluções que minimizem os efeitos causados pelos transtornos alimentares.

Agradecimento: À professora Ana Tereza Guimarães responsável pela análise estatística do estudo. 
Pires R, Pinto J, Santos G, Santos S, Zraik H, Torres L, Ramos M. Rastreamento da freqüência de comportamentos sugestivos de transtornos alimentares na Universidade Positivo. Rev Medicina (São Paulo). 2010 abr.-jun.;89(2):115-23.

\begin{abstract}
Objective: The aim of this report is to trace the frequency of behavior that suggest eating disorders in a group of students of courses which were previously randomly selected among those of Universidade Positivo and compare the prevalence of these disorders between the courses and between men and women, confronting these data with those in worldwide literature. Methods: This is a prevalence study in which the auto- scales of Bite (Bulimia Edimburgh Investigation Test) and EAT-26 (Eating Attitude Test) were applied in 463 university students of biological and health courses in Universidade Positivo, who had been previously selected at random. Results: According to the scale of EAT-26, 23 students (5\%) presented possible eating disorder, with a significant predominance of females. Moreover, the autoscale of BITE indicated that 17 students (4\%) had a score compatible with nervous bulimia. It has also been found, according to the BITE-severity scale, that 6 students (1\%) had very severe disorder and 33 students $(7,3 \%)$ had a score that suggested a compromised clinical state. Conclusions: This study shows that the frequency of eating disorders in the population studied, as well as the frequency of inadequate eating behavior, particularly among females, is similar to the data available in literature. It is possible to conclude that these findings are relevant to the clinical practice among the age that was studied and that they can lead to the development of assistencial measures for patients with eating disorders and to future studies in which we might be able to have better understanding of the risk factors that contribute to the development of eating disorders.
\end{abstract}

KEY WORDS: Anorexia; Bulimia; Eating disorders; Cross-sectional studies; Students, health occupations; Risk factors.

\title{
REFERÊNCIAS
}

1. Pinto ACM, Camargo MR, Novo NF, Hübner, CK. Transtornos alimentares em alunas da Faculdade de Medicina do Centro de Ciências Médicas e Biológicas da PUC-SP. Rev Fac Cienc Med Sorocaba. 2009;11:16-20.

2. Herzog DB, Sacks NR, Keller MB, Lavori PW, Ranson $\mathrm{KB}$, Gray HM. Patterns and predictors of recovery in anorexia nervosa and bulimia nervosa. J Am Acad Child Adolesc Psychiatry. 1993;32:835-42.

3. Mahan LK, Stump SE. Nutrição na adolescência. In: Mahan KL, Escott-Stump S, editores. Krause alimentos, nutrição e dietoterapia. 9a ed. São Paulo: Roca; 1998. p.279-83.

4. Fisher M, Golden NH, Katzman DK, Kreipe RE, Ress J, Schebendach J, et al. Eating disorders in adolescents: a background paper. J Adolesc Health. 1995;16:420-37.

5. Cenci M, Peres KG, Vasconcelos FAG. Prevalência de comportamento bulímico e fatores associados em universitárias. Rev Psiq Clin. 2009;36(3):83-8.

6. Abott DW, Ackerman SH, Agras WS, Banzhaf D, Barber J, Bartlett JC, et al. Practice guideline for eating disorders. Am J Psychiatry. 1993;150:212-24.

7. Doyle J, Bryant-Waugh R. Epidemiology. In: Lask B, Bryant-Waugh R, editors. Anorexia nervosa and related eating disorders in childhood and adolescence. 2th ed. East Sussex: Psychology Press; 2000. p. 41-61.
8. American Psychiatry Association. Practice guideline treatment for psychiatric disorders: compendium 2000. Washington (DC): The Association; 2000.

9. Jones JM, Bennett S, Olmsted MP, Lawson L, Rodin G. Disordered eating attitudes and behaviours in teenaged girls: a school-based study. CMAJ. 2001;165:547-52.

10. Melin $P$, Araújo AM. Transtornos alimentares em homens: um desafio diagnóstico. Rev Bras Psiquiatr. 2002;24(3):73-6.

11. Yates A. Current perspectives on the eating disorders: I. history, psychological and biological aspects. J Am Acad Child Adolesc Psychiatry. 1989;28(6):813-28.

12. Drewnowski A, Yee DK, Kurth CL, Krahn DD. Bulimia in college women: incidence and recovery rates. Am J Psychiatry. 1988;145:753-5.

13. Katzman M, Wolchik S, Braver T. The prevalence of frequent binge-eating and bulimia in a non-clinical college sample. Int J Eat Disord. 1984;3:53-62.

14. Pyle RL, Halvorson PA, Newman PA, Golff GM. The incidence of bulimia in freshman college students. Int J Eat Disord. 1983;2:75-85.

15. Halmi KA, Falk JR, Schwartz E. Binge-eating and vomiting: a survey of a college population. Psychol 
Med. 1981;11:697-706.

16. Hart KJ, Ollendick TH. Prevalence of bulimia in working and university women. Am J Psychiatry. 1985;142:851-4.

17. Herzog DB, Pepose M, Norman DK, Rigotte MA. Eating disorders and social maladjustment in female medical students. J Nerv Ment Dis. 1985;173:734-7.

18. Freitas S, Gorenstein C, Appolinario JC. Instrumento para avaliação dos transtornos alimentares. Rev Bras Psiquiatr. 2002;24(S3):34-8.

19. Lewinsohn PM, Striegel-Moore RH, Seeley JR. Epidemiology and natural course of eating disorders in young women from adolescence to young adulthood. J Am Acad Child Adolesc Psychiatry. 2000;39:128492.

20. Henderson M, Freeman CPL. A self-rating scale for bulimia. The BITE. Br J Psychiatry. 1987;150:18-84.

21. Cordas TA, Hochgraf PB. O BITE. Instrumento para avaliação de bulimia nervosa - versão para o português. J Bras Psiquiatr. 1993;42:141-4.

22. Gross J, Rosen JC, L Eitenberg, H.; Willmuth, ME. Validity of the eating attitudes test and The Eating Disorders Inventory in Bulimia Nervosa. J Consult Clin Psychol. 1986;54:875-6.

23. Nunes MA, Bagatini LF, Abuchaim AL, Kung A, Ramos D, Silva JA, Someuzi L, Pinheiro A. Distúrbios da conduta alimentar: considerações sobre o Teste de Atitudes Alimentares (EAT). Rev ABP-APAL. 1994;16:7-11.

24. Cordás TA, Neves JEP. Escalas de Avaliação em transtornos alimentares. Rev Psiquiatr Clin. 1999;26 (1):41-7.

25. Garfinkel PE, Newman A. The eating attitudes test: twenty-five years later. Eat Weight Disord. 2001;6:124.

26. Mehler PS. Bulimia nervosa. N Engl J Med. 2003;349:9.

27. Souza FGM, Martins MCR, Monteiro FCC, Menezes Neto
GC, Ribeiro IB. Anorexia e bulimia nervosa em alunas da Faculdade de Medicina da Universidade Federal do Ceará - UFC. Rev Psiq Clin. 2002;29(4):172-80.

28. Vilela JEM, Lamounier JA, Dellaretti Filho MA, Barros Neto JR, Horta GM. Transtornos alimentares em escolares. J Pediatria. 2004;80 (1):49-54.

29. Dunker KLL, Fernandes CPB, Carreira Filho D. Influência do nível socioeconômico sobre comportamentos de risco para transtornos alimentares em adolescentes. J Bras Psiquiatr. 2009;58(3):156-61.

30. Drewnowski A, Yee DK, Krrahn DD. Bulimia in College Women. Am J Psychiatry. 1988;145:753-5.

31. Katzman M, Wolchik S, Braver T. The Prevalence of Frequent Binge Eating and Bulimia in a Non-clinical College Sample. Int J Eating Disord. 1984;3:53-62.

32. Pyle RL, Halvorson PA, Newman PA, Golff GM. The incidence of bulimia in Freshman College Students. Int J Eating Disord. 1983;2:75-85.

33. Albuquerque MR, Souza PS, Luz SS. Avaliação dos sintomas de transtornos alimentares, imagem corporal e nível de atividade física em alunos de uma universidade pública do interior de Minas Gerais. In: 21ํㅡㄹ Congresso Internacional de Educação Física (FIEP); 2006.

34. Nunes MA, Olinto MTA, Barros FC, Camey S. Influência da percepção do peso e do índice de massa corporal nos comportamentos alimentares anormais. Rev Bras Psiquiatr. 2001;23(1):21-7.

35. Ghazal N, Agoub M, Moussaoui D, Battas O. Prevalence of bulimia among secondary school students in Casablanca. Encephale. 2001;27(4):338-42.

36. Miotto P, De Coppi M, Frezza M, Preti A. Eating disorders and suicide risk factors in adolescents: an Italian community-based study. J Nerv Ment Dis. 2003;191(7):337-43.

37. Luz SS, Eufrásio AS, Souza JMP, Philippi ST. Variáveis associadas à presença de sintomas de transtornos alimentares em universitários de Belo Horizonte, Brasil. In: XIV Congresso Latino-Americano de Nutrição (SLAN); 2006. 